\title{
Evaluation of Soybean Germplasm Lines for Agro-Morphological Traits and Terminal Drought Tolerance
}

\author{
V. Sreenivasa ${ }^{1}$, S.K. Lal ${ }^{2}$, A. Talukdar ${ }^{2}$, P. Kiran Babu ${ }^{3}$, \\ H.K. Mahadeva Swamy ${ }^{1}$, Darsing R. Rathod ${ }^{2}$, Raju R. Yadav, \\ Shatakashi Poonia ${ }^{2}$, K.V. Bhat ${ }^{3}$ and C. Viswanathan ${ }^{4}$ \\ ${ }^{1}$ Division of crop Improvement, ICAR-Sugarcane Breeding institute, Coimbatore, India \\ ${ }^{2}$ Division of Genetics, ICAR-Indian Agricultural Research Institute, New Delhi, India \\ ${ }^{3}$ Division of Plant Genetic Resources, ICAR-National Bureau of Plant Genetic Resources, \\ New Delhi, India \\ ${ }^{4}$ Division of Plant Physiology, ICAR-Indian Agricultural Research Institute, New Delhi, India \\ *Corresponding author
}

\section{A B S T R A C T}

\begin{tabular}{l} 
K e y w o r d s \\
$\begin{array}{l}\text { Terminal, } \\
\text { Germplasm } \\
\text { accessions, } \\
\text { Potassium Iodide } \\
\text { (KI), Soybean, } \\
\text { drought tolerance }\end{array}$ \\
\hline Article Info \\
\hline $\begin{array}{l}\text { Accepted: } \\
\text { 04 March } 2019 \\
\text { Available Online: } \\
\text { 10 April } 2019\end{array}$ \\
\hline
\end{tabular}

\section{Keywords}

Terminal, Germplasm accessions, Potassium Iodide (KI), Soybean, drought tolerance

The occurrence of drought stress at seed filling stage is known to cause severe yield reduction in soybean especially where the crop is grown in rainfed conditions. Screening of large germplasm lines under natural drought conditions is extremely difficult to execute due to unusual rains. In the present study, about 328 germplasm lines are screened for terminal drought tolerance by spraying $0.2 \%$ of potassium iodide $(\mathrm{KI})$ at $\mathrm{R}_{5}$ stage and the tolerant lines were again retested under similar conditions in the subsequent year. The lines were classified as tolerant, moderately tolerant and susceptible based on the relative reduction in seed yield and 100-seed weight of treated over control conditions. The Shannon diversity index (SDI) indicated that genotypes were highly diverse for seed colour $\left(\mathrm{H}^{\mathrm{I}}=1.20\right)$ and hilum colour $\left(\mathrm{H}^{\mathrm{I}}=0.93\right)$. The PCA biplot analysis revealed that lines were more compactly and closely placed under controlled conditions as against treated. Four genotypes (TGX1835-3E, VSL-69, EC-105780 and PK-1243) were identified as relatively drought tolerant lines as they showed less reduction for number of pods per plant, seed yield and hundred seed weight under KI induced drought conditions. These lines were again validated next year and were found to be potential source for the development of drought tolerant varieties for the sustainable soybean production.

\section{Introduction}

Soybean (Glycine max L. (Merrill) is one of the leading oilseed crops grown for its edible oil and protein in India as well as world over. Soybean seed contains over $40 \%$ protein and
$20 \%$ oil and $35 \%$ carbohydrate (Liu et al., 1997) and its milk is considered as important source of food to infants in china. Recent studies have indicated that consumption of soybean reduces cancer, blood serum cholesterol, osteoporosis and heart disease 
(Birt et al., 2004). It is also a good source of minerals, vitamins, folic acid and iso-flavones which are credited with slow development of these diseases (Wilson et al., 2004). In India more than $90 \%$ of soybean area is under rainfed conditions and the frequency of droughts is common phenomenon. Drought is one of the single most factors responsible for more than $50 \%$ reduction in soybean yields (Boyer et al., 1982; Bray et al., 2000). Soybean cultivation in India is overly dependent on seasonal monsoon rains which are erratic and uneven, causing termination of growth from germination to seed filling (Joshi and Bhatia, 2003). Drought is known to affect soybean yield by affecting all stages of plant growth and development; from germination to flowering, and seed filling to development as well as seed quality (Siddique et al., 2001; Manavalan et al., 2009). Occurrence of drought stress during vegetative stage can be compensated with rains during later part of crop growth, however drought at terminal growth stage especially during seed filling to seed maturity stage would cause severe yield loss which could not be recovered by any means (Sionit and Kramer, 1977; Hirasawa et al., 1994; Saitoh et al., 1999). Terminal drought stress in soybean causes gradual reduction in photosynthetic rate, followed by senescence of leaves and reduced seed size that finally results in reduced grain yields (Brevedan and Egli, 2003; Manavalan et al., 2009). Reduced photosynthetic rate affects the synthesis and transportation of photosynthates from leaf to the seed causing reduction in seed size. However photosynthates stored in stem acts as reserves plays a pivotal role in substituting factor for seed filling and seed development in soybean (Constable and Hearn, 1978). Photosynthates stored in stem acts as an alternate source for seed development at times of terminal drought stress (Schnyder, 1993; Subbarao et al., 1995). In soybean, it has been reported that about $25 \%$ of seed weight is obtained from stem reserves (Constable and Hearn, 1978).

One of the most sustainable ways to overcome the recurring and perennial problem of drought and to make soybean production more stable and sustainable is to develop climate resilient soybean genotypes with relatively drought tolerant to tide over short periods of drought stress at seed filling stage. Yield losses could be greatly reduced by identifying and adopting drought tolerant genotypes. However, no systematic breeding efforts for developing drought tolerant soybean genotypes are limiting due to the lack of proper and reliable field screening techniques. Field screening of large germplasm lines by withholding irrigation facility at particular stage is rather more cumbersome and time consuming as well as difficult execute due to monsoon rains. Few techniques were developed and standardized to stimulate drought like conditions under field conditions with the application of chemicals. Various indices/parameters have been adopted to quantify drought tolerance in soybean genotypes and other crops ( $\mathrm{Ku}$ et al., 2013). Potassium Iodide (KI) is known to mimic drought stress under natural conditions, it acts as desiccant on plants by reducing photosynthetic rate, chlorophyll content and senescence with increased content of sucrose and proline content (Sawhney and Singh, 2002) and the effect of drought stress on seed weight reduction could be compared with that of natural drought stress conditions. A single spray of $\mathrm{KI}$ at reproductive stage especially during seed filing stage (Blum et al., 1983a; Bouslama et al., 1984; Regan et al., 1993) helps in differentiating genotypes based on their ability to form viable seeds and this method of screening is used to evaluate large number of germplasm lines for terminal drought tolerance traits in many crops (Nicolas and Turner, 1993; Royo and Blanco, 1998, Ashraf et al., 2003; Singh et al., 2012). 
The present study was carried out to identify soybean lines tolerance to terminal drought tolerance.

Lack of progress in the development of drought tolerant varieties in soybean is mainly attributed to non-availability of proper screening facilities, poor understanding of physiological and biochemical responses of soybean varieties to drought (Bhatia et al., 2014). Keeping these potential research gaps in view, the present investigation was formulated to evaluate the soybean germplasm for agro-morphological traits and terminal drought tolerance induced by $\mathrm{KI}$ under the field conditions. The main objectives of this study were (i) Evaluation of soybean germplasm lines for agromorphological traits (ii) screening for terminal drought tolerance using KI (Potassium Iodide) and (iii) Identifying the soybean genotypes for drought tolerance.

\section{Materials and Methods}

\section{Experimental site and weather conditions}

The experiment was laid out at India Council of Agricultural Research-Indian Agricultural Research Institute (ICAR-IARI), New Delhi, India. The experimental farm has sandy loam to loamy soil with $\mathrm{pH}$ of 7.5 having semi-arid subtropical climate with an average temperature ranging from 19 to $32^{\circ} \mathrm{C}$ (July to November).

\section{Experimental material and field evaluation}

The experimental material consists of a 328 soybean germplasm lines (Table 1) selected randomly from Germplasm Management Unit at Division of Genetics, ICAR-IARI, Pusa Campus, New Delhi. Each accession was planted in two rows of three meter length, sown during $1^{\text {st }}$ week of July 2014 in an augmented block design (Federer 1956) along with five checks varieties viz., Pusa 9712, SL 688, PS 1347 Ps 1092 and Bragg. The checks were replicated once after every 10 germplasm lines. The recommended row-torow and plant-to-plant spacing of 45 and $5 \mathrm{~cm}$ respectively was followed and all the agronomic practices were carried out timely to raise a healthy crop. The crop was raised by providing regular irrigation facilities without any biotic or abiotic stress symptoms until the seed filling stage $\left(\mathrm{R}_{5}\right)$. At $\mathrm{R}_{5}$ stage the plants of one replication was sprayed/drenched completely with $0.2 \%$ of Potassium Iodide (KI) to mimic terminal drought stress (Bhatia et al., 2014). Severity of terminal drought on germplasm lines was measured based on percent reduction of seed yield and 100-seed weight in treated as against normal was calculated and genotypes were grouped in to three different classes viz., Tolerant $(0-20 \%)$, Moderately susceptible $(20.1-45 \%)$, susceptible $(45.1-70 \%)$ as described by Bhatia et al., (2014). The following traits were recorded from five randomly selected plants from each genotype of both control and treated plots and mean values were computed for analysis purposes. The quantitative traits were Days to 50 per cent flowering (DFF), Days to full maturity (DFM), Plant height (PH), Number of seeds per pod (NSP), Number of pods per plant (NPP), Hundred seed yield (HSW), Single plant yield (SPY) and Row yield (RY). Ten qualitative traits were recorded at flowering stage was growth habit, leaf shape, flower color, pod color, pod pubescence, pubescence color, seed shape, seed color, seed luster and hilum color. During second season (2015), 40 genotypes were chosen based on first year (2014) field screening results in such a way that equal number of lines from tolerant, moderately susceptible and susceptible lines for terminal drought tolerance trait and five check varieties were planted in a randomized block design consisting of two replications (Table 1b). One replication was imposed 
terminal drought stress at $\mathrm{R}_{5}$ stage by drenching $\mathrm{KI}$ at $0.2 \%$ and the lines were screened for drought tolerance by recording seed yield and its contributing traits. Diversity parameters were calculated for qualitative traits by taking account of allelic richness (calculated from descriptor states) and allelic evenness through Shannon Diversity Index (SDI) (Shanon and Weaver, 1949) as follows:

$\mathrm{SDIi}=\sum_{\mathrm{i}=1}^{\mathrm{di}} \mathrm{Pij} . \log \mathrm{Pij}_{\mathrm{ij}}$

Where $\mathrm{SDI}_{\mathrm{i}}=\mathrm{SDI}$ for $\mathrm{i}^{\text {th }}$ descriptor, $\mathrm{di}=$ descriptor state for $i^{\text {th }}$ descriptor, pij $=$ the proportion of accessions for $j^{\text {th }}$ descriptor states of $i^{\text {th }}$ descriptor. Analysis of variances (ANOVA) was carried out using SAS 9.3 software (SAS Institute Inc., Cary, NC, USA). Estimate of co-efficient of variation $(\mathrm{CV})$ was calculated as per the standard formulae (Burton 1952) and expressed in percent. Principal Components Analysis (PCA) was done using XL stat.

\section{Results and Discussion}

\section{Diversity analysis}

The Shannon diversity index (SDI) for 10 qualitative traits of the 328 germplasms lines were presented in Table 4. The highest SDI observed for seed colour (1.20) and lowest for pod pubescence $(0.13)$ and with a mean of 0.57. This indicates that germplasms are highly diverse for seed colour $\left(\mathrm{H}^{\prime}=1.20\right)$ and hilum colour $\left(\mathrm{H}^{\prime}=0.93\right)$, whereas it is lowest for pod pubescence $\left(\mathrm{H}^{\prime}=0.13\right)$, pod colour $\left(H^{\prime}=0.21\right)$ and leaf shape $\left(H^{\prime}=0.25\right)$. Gangopadhyay et al., (2016) reported high species diversity for intensity of stem colour, leaf shape, epicalyx shape, 13 quantitative characters and 3 biotic stress parameters between wild and cultivated species of $O \mathrm{kra}$ using SDI. In another study, Upadhyaya et al., (2001) reported that SDI was used to classify chickpea core collection accessions into desi, intermediate and kabuli types based on 7 morphological descriptors and 15 agronomic characters.

\section{Evaluation accessions for agro- morphological characters}

Descriptive statistics for 328 genotypes under control and treated conditions were presented in Table 2c. The analysis of variance for control and treatment plots during 2014 season has revealed significant differences $(p<0.05)$ for all morphological traits except for 100-seed weight (HSW) under control conditions and number of seeds per pod (NSPP) as well as single plant yield (SPY) under control and treated conditions (Table $2 \mathrm{a} \& \mathrm{~b})$. This indicates that there is sufficient quantity of variability present in the germplasm lines included in the present study for quantitative traits. The coefficient of variation ranged from $2.43 \%$ (days to $50 \%$ flowering) to $18.76 \%$ (row yield) under normal conditions where as under KI treated conditions it varied from $2.61 \%$ (days to $50 \%$ flowering) to $25.58 \%$ (single plant yield). Whereas during second season (2015) all the traits number of seeds per pod (NSPP) under control conditions, exhibited significant differences $(\mathrm{p}<0.05)$ for all quantitative traits. The random sample selected based on the first season results do possess much variability for all the characters. Coefficient of variation varied from 1.33 (Days to full maturity) to 47.62 (number of seeds per pod) under controlled conditions, whereas it was 5.31 (Plant height) to 24.55 (number of pods per plant) under KI treated conditions (Table $3 a \& b)$.

\section{Principle component analysis}

Terminal drought occurs when plants are exposed to water deficit during later stages of crop growth especially seed filling and seed 
development. The greatest loss occurs when the drought occurs during flowering stage (Frahm et al., 2004). The effect of drought on the plant and its reactions depend also on duration and intensity of stress (Brar et al., 1990). Principal components (PCs) and Eigen values of quantitative traits were analyzed to determine percent contribution of individual trait to the diversity under both the conditions is presented in Table $4 a$ and $b$. Based on Eigen value of more than 1, PC1 and PC2 together explained $64.80 \%$ variation and $61.71 \%$ variation under controlled and treated conditions respectively. To be specific PC1 alone accounted $51.23 \%$ and $46.77 \%$ of variance under normal and treated conditions respectively. Remaining components contributed $35.20 \%$ under control and $38.23 \%$ under treated conditions to total diversity. The traits 100-seed weight, number of pods/plant, no of seeds/pod, row yield and single plant yield had major contributions towards PC1 and for $\mathrm{PC} 2$ the maximum variation contributed by plant height, number of seeds per plant, Days to $50 \%$ flowering, Days to full maturity, hundred seed weight and single plant yield under control conditions (Table 5c). Under KI treated conditions highest variation in PC1 was contributed by hundred seed weight, number of pods per plant, number of seeds per plant, row yield and single plant yield and in case of PC2, the main traits which contributed to maximum variation was days to $50 \%$ flowering, days to full maturity, plant height, single plant yield, number of seeds per pod and hundred seed weight. Thus under both conditions PC1 is mainly related to economic yield traits, whereas PC2 for other vegetative traits. Similar results were found by Deepika et al., (2017) and Iqbal et al., (2008) in Soybean and Gangopadhyay et al., (2016) in okra. The PCA Biplot analysis indicated that genotypes were highly clustered and closely placed under control condition as compared with the treated conditions, they were loosely arranged due to the differential response of genotypes towards KI induced terminal drought stress (Fig. 1a \& b). PC1 and PC2 having Eigen values higher than unity explained $82.55 \%$ of total variability among soybean genotypes attributed to seed yield (El-Hashash et al., 2016). In another study, Kargar et al., (2015) stated that, PC1, PC2 and PC3 contributed $32.57 \%, 27.20 \%$ and $15.02 \%$ to total variability and also explained $74.79 \%$ of traits variation with varimax rotation method under stress condition. Mahbub et al., (2016) has indicated that, the PCA yield four of the Eigen values above unity accounted for $91.55 \%$ of the total variation. The first three principles accounted for $83.23 \%$ of the total variation. In the table $5 \mathrm{a}, \mathrm{PC} 1$ has highest positive load from single plant yield (17.22), row yield (15.93), number of pods per plant (15.58), days to full maturity (15.39) and days to $50 \%$ flowering (14.06) under normal conditions, but under KI treated conditions (Table 5b) single plant yield (18.75), number of pods per plant (18.29), days to full maturity (17.17) and row yield (16.30) has highest contribution to the total diversity. Quantitative traits contributed positively to first three principal components and hence these could be given considerable importance for the genetic material under investigation by Iqbal et al., (2008).

\section{Screening of germplasm lines for terminal drought tolerance using KI}

Presence of genetic variation for physiological traits is of the pivotal importance for the development of varieties for drought tolerance. Differential response of genotypes towards abiotic factors makes screening easy and effective in identification of drought tolerance genotypes. Lines expressing higher degree of tolerance towards terminal drought is characterized by their capacity in partition of photosynthates to the developing seeds after stress was imposed 
(Nicolas and Turner, 1993; Ashraf et al., 2003; Singh et al., 2012). The faster rate of seed filling in few lines under KI treated conditions might have played a role in their ability to produce bigger seeds and higher seed yields that intern resulted in relative tolerance to terminal drought. In the present study, 328 germplasm lines were evaluated to identify terminal drought tolerant lines by the application of KI spray at $\mathrm{R}_{5}$ stage to create drought like situation. The mean seed yield per row under control conditions was 175.57 $\mathrm{g}$ with a range of 6 to $634.0 \mathrm{~g}$, whereas under KI treated conditions the mean yield was $67.88 \mathrm{~g}$ with the range of 2.0 to $308.0 \mathrm{~g}$ (Table 6). The mean seed yield reduction is $61.50 \%$ under KI treated drought conditions as compared to control. The seed yield reduction among the entries varies from $1.94 \%$ to a as high as $97.44 \%$. For seed yield per plant, the average under control is $8.86 \mathrm{~g}$, whereas under treated conditions it is $4.26 \mathrm{~g}$, the reduction under treated conditions varies from $0.54 \%$ to the highest of $95.01 \%$. The mean hundred seed weight is $8.86 \mathrm{~g}$ and 4.26 $\mathrm{g}$ under control and treated conditions respectively. The average reduction for hundred seed weight is $33.41 \%$ under treated as compared with control conditions and the range of reduction varies from $0.62 \%$ to $96.67 \%$. For pods per plant, the mean is $62.10 \%$ under control conditions, whereas it is $40.54 \%$ treated conditions. The average reduction for number of pods is $0.78 \%$ to $87.34 \%$. The results have clearly indicated that there are large genotypic variations in response to KI spray induced drought stress in soybean germplasm lines. The lines were grouped into three different classes based on the percent reduction in seed yield and hundred seed weight of treated plots over control (Table 7). The genotypes,TGX18353E, VSL-61 and PK-1243 and EC-105790 has lowest $(<16.59 \%)$ reduction for seed yield and hundred seed weight under treated conditions and are considered as relatively tolerant to KI induced terminal drought stress, another group of lines consisting of G-2130, DS-9802, SL-633 and PKS-25 has a recorded 20.1 to $45 \%$ reduction for seed yield and hundred seed weight, whereas PK-1024, PK1240, DS-2309 and UPSL-291 recorded the highest reduction $(>50 \%)$ for seed yield and hundred seed weight under KI induced drought conditions.

\section{Validation of identified drought tolerant lines for yield traits under field conditions}

Performance of genotypes under KI induced terminal drought tolerance is verified further during next season (2015) by evaluating 40 genotypes based on first year results. Most genotypes showed consistent and similar responses like first year for all quantitative traits under KI induced terminal drought tolerance. Four terminal drought tolerant lines were identified during first year viz., TGX1835-3E, VSL-69, EC-105890 and PK1243 were evaluated in second year for seed yield contributing traits under KI induced drought tolerance is discussed (Table 8). The average seed yield reduction ranged from $8.57 \%$ (EC-1055780) to $16.19 \%$ (PK-1243) under KI treated as compared to control. Seed yield per plant recorded $15.79 \%$ in EC105780 to $19.59 \%$ in TRX1835-3E under treated over control. For hundred seed weight, the reduction is less than $10 \%$ across the four lines. From the above analysis, the lines (TGX1835-3E, VSL-69, EC-105780 and PK1243) were identified as relatively drought tolerant in Kharif-2014 based on seed yield and hundred seed weight and had shown less than $20 \%$ reduction under treated conditions in the next season. The tolerant genotypes exhibited the capacity to produce healthy and normal seeds due to their ability to store the photosynthates in stem and translocate them to the developing seeds during drought like condition made them produce relatively good yields. 
Table.1 List of soybean genotypes and their origin used for screening against terminal drought using KI during Kharif-2014

\begin{tabular}{|c|c|c|c|c|c|}
\hline S. No & Genotype & origin & S. No & Genotype & Origin \\
\hline 1 & BS-1 & BIRSA, Ranchi & 44 & G-2132 & AVRDC \\
\hline 2 & BJJF-8 & Unknown & 45 & IC-101449 & Indigenous collection \\
\hline 3 & DS 74 & DS-Delhi & 46 & IC-141446 & Indigenous collection \\
\hline 4 & DS-76-1-3 & DS-Delhi & 47 & JS-91-4 & Jabalpur \\
\hline 5 & DS-76-1-2-2 & DS-Delhi & 48 & JS(SH)91-16 & sehore,MP \\
\hline 6 & DS-76-1-2-3 & DS-Delhi & 49 & KALITUR & Indigenous collection \\
\hline 7 & DS-MM-64 & DS-Delhi & 50 & KG-83-1A & Kasbe-Digraj \\
\hline 8 & DS-93-108A & DS-Delhi & 51 & KB-17 & Kasbe-Digraj \\
\hline 9 & DS-9703 & DS-Delhi & 52 & L-377 & AVRDC \\
\hline 10 & DS-9802 & DS-Delhi & 53 & L-416 & AVRDC \\
\hline 11 & EC-109514 & EC-exotic & 54 & $\mathrm{~L}-440$ & AVRDC \\
\hline 12 & EC-109565 & EC-exotic & 55 & L-587 & AVRDC \\
\hline 13 & EC-113396 & EC-exotic & 56 & L-652 & AVRDC \\
\hline 14 & EC-114526 & EC-exotic & 57 & L-680 & AVRDC \\
\hline 15 & EC-25720 & EC-exotic & 58 & M-135 & AVRDC \\
\hline 16 & EC-76758 & EC-exotic & 59 & M-53 & AVRDC \\
\hline 17 & EC-389148 & EC-exotic & 60 & M-11913 & AVRDC \\
\hline 18 & EC-389179 & EC-exotic & 61 & M-693 & AVRDC \\
\hline 19 & EC-389392 & EC-exotic & 62 & MACS-57 & Pune \\
\hline 20 & EC-439618 & EC-exotic & 63 & MAUS-71 & Parbhani \\
\hline 21 & EC-439619 & EC-exotic & 64 & MAUS-311 & Parbhani \\
\hline 22 & EC-14436 & EC-exotic & 65 & NRC 37 & Indore \\
\hline 23 & EC-39779 & EC-exotic & 66 & PK-1162 & Pantnagar \\
\hline 24 & EC-39873 & EC-exotic & 67 & PK-564 & Pantnagar \\
\hline 25 & EC-97351 & EC-exotic & 68 & PK-1023 & Pantnagar \\
\hline 26 & EC-105790 & EC-exotic & 69 & PK-1169 & Pantnagar \\
\hline 27 & EC-95299 & EC-exotic & 70 & PK-1180 & Pantnagar \\
\hline 28 & G-395 & AVRDC & 71 & PK-1223 & Pantnagar \\
\hline 29 & DS-12-13 & DS-Delhi & 72 & PK-1240 & Pantnagar \\
\hline 30 & G-2253 & AVRDC & 73 & PK-1243 & Pantnagar \\
\hline 31 & G-2265 & AVRDC & 74 & PK-1250 & Pantnagar \\
\hline 32 & G-2344 & AVRDC & 75 & PK-295 & Pantnagar \\
\hline 33 & G-2601 & AVRDC & 76 & PLSO-6A & Pantnagar \\
\hline 34 & G-2602 & AVRDC & 77 & PLOS-91 & Pantnagar \\
\hline 35 & G-2603 & AVRDC & 78 & SL-46 & PAU, Ludhiana \\
\hline 36 & G-2608 & AVRDC & 79 & SL-432 & PAU, Ludhiana \\
\hline 37 & DS 2614 & DS-Delhi & 80 & SL-443 & PAU, Ludhiana \\
\hline 38 & G-2631 & AVRDC & 81 & SL-459 & PAU, Ludhiana \\
\hline 39 & G-2650 & AVRDC & 82 & SL-284 & PAU, Ludhiana \\
\hline 40 & G-2651 & AVRDC & 83 & TGX1828-4E & IITA, Nigeria \\
\hline 41 & G-2656 & AVRDC & 84 & TGX1831-32E & IITA, Nigeria \\
\hline 42 & G-2670 & AVRDC & 85 & TGX1864-25F & IITA, Nigeria \\
\hline 43 & G-2130 & AVRDC & 86 & TGX1835-3E & IITA, Nigeria \\
\hline
\end{tabular}


Table.1 List of soybean genotypes and their origin used for screening against terminal drought using KI during Kharif-2014

\begin{tabular}{|c|c|c|c|c|c|}
\hline S. No & Genotype & origin & S. No & Genotype & origin \\
\hline 87 & TGX1973-14 & IITA, Nigeria & 130 & UPSV-22 & Pantnagar \\
\hline 88 & TGX1019-2FB & IITA, Nigeria & 131 & UPSV 24 & Pantnagar \\
\hline 89 & TAMS-38 & BARC-amravati & 132 & UPSV-65A & Pantnagar \\
\hline 90 & UGM-47 & AVRDC & 133 & UPSV-72 & Pantnagar \\
\hline 91 & UGM-77 & AVRDC & 134 & UPSV-19 & Pantnagar \\
\hline 92 & UPSL-19 & Pantnagar & 135 & UPSV-31 & Pantnagar \\
\hline 93 & UPSL-34 & Pantnagar & 136 & VLS-17 & VPKS, Almora \\
\hline 94 & UPSL-54 & Pantnagar & 137 & EC-439597 & EC-exotic \\
\hline 95 & UPSL-62 & Pantnagar & 138 & EC-439606 & EC-exotic \\
\hline 96 & UPSL-64 & Pantnagar & 139 & EC-389170 & EC-exotic \\
\hline 97 & UPSL-92 & Pantnagar & 140 & EC389116 & EC-exotic \\
\hline 98 & UPSL-152 & Pantnagar & 141 & EC458355 & EC-exotic \\
\hline 99 & UPSL-156-B & Pantnagar & 142 & EC-458383 & EC-exotic \\
\hline 100 & UPSL-162 & Pantnagar & 143 & EC-456525 & EC-exotic \\
\hline 101 & UPSL-163 & Pantnagar & 144 & SL 525 & PAU, Ludhiana \\
\hline 102 & UPSL-180 & Pantnagar & 145 & EC-456616 & EC-exotic \\
\hline 103 & UPSL-181 & Pantnagar & 146 & EC-456639 & EC-exotic \\
\hline 104 & UPSL-211 & Pantnagar & 147 & EC-457772 & EC-exotic \\
\hline 105 & SL-525 & PAU, Ludhiana & 148 & EC-471315 & EC-exotic \\
\hline 106 & UPSL-291 & Pantnagar & 149 & EC-471319 & EC-exotic \\
\hline 107 & UPSL-298 & Pantnagar & 150 & EC-471851 & EC-exotic \\
\hline 108 & UPSL-309 & Pantnagar & 151 & EC-471853 & EC-exotic \\
\hline 109 & UPSL-326 & Pantnagar & 152 & EC-471870 & EC-exotic \\
\hline 110 & UPSL-332-B & Pantnagar & 153 & EC-471881 & EC-exotic \\
\hline 111 & UPSL-340-B & Pantnagar & 154 & EC-471882 & EC-exotic \\
\hline 112 & UPSL-343 & Pantnagar & 155 & EC-471909 & EC-exotic \\
\hline 113 & UPSL-505 & Pantnagar & 156 & EC-471910 & EC-exotic \\
\hline 114 & UPSL-652 & Pantnagar & 157 & EC-471920 & EC-exotic \\
\hline 115 & UPSL-656 & Pantnagar & 158 & EC-471921 & EC-exotic \\
\hline 116 & UPSL-706 & Pantnagar & 159 & EC-471936 & EC-exotic \\
\hline 117 & UPSL-736 & Pantnagar & 160 & EC-471-937 & EC-exotic \\
\hline 118 & UPSL-558 & Pantnagar & 161 & EC-471956 & EC-exotic \\
\hline 119 & UPSL-769 & Pantnagar & 162 & EC-471967 & EC-exotic \\
\hline 120 & UPSL-782 & Pantnagar & 163 & EC-471969 & EC-exotic \\
\hline 121 & UPSL-784 & Pantnagar & 164 & EC-471972 & EC-exotic \\
\hline 122 & UPSL-785 & Pantnagar & 165 & EC-471979 & EC-exotic \\
\hline 123 & UPSL-786 & Pantnagar & 166 & EC-471981 & EC-exotic \\
\hline 124 & UPSL-787 & Pantnagar & 167 & EC-472119 & EC-exotic \\
\hline 125 & UPSL-57 & Pantnagar & 168 & EC-472120 & EC-exotic \\
\hline 126 & UPSM-595 & Pantnagar & 169 & EC-472162 & EC-exotic \\
\hline 127 & UPSV-2 & Pantnagar & 170 & EC-472173 & EC-exotic \\
\hline 128 & UPSV-19 & Pantnagar & 171 & EC-475184 & EC-exotic \\
\hline 129 & SL 525 & PAU, Ludhiana & 172 & EC-472197 & EC-exotic \\
\hline
\end{tabular}


Table.1 List of soybean genotypes and their origin used for screening against terminal drought using KI during Kharif-2014

\begin{tabular}{|c|c|c|c|c|c|}
\hline S. No & Genotype & origin & S. No & Genotype & origin \\
\hline 173 & EC-472199 & EC-exotic & 216 & PK-1251 & Pantnagar \\
\hline 174 & EC-472202 & EC-exotic & 217 & PK-1259 & Pantnagar \\
\hline 175 & EC-472203 & EC-exotic & 218 & PK-1274 & Pantnagar \\
\hline 176 & EC-471720 & EC-exotic & 219 & PK-1283 & Pantnagar \\
\hline 177 & EC-457191 & EC-exotic & 220 & 19-P3 & Indore \\
\hline 178 & RKS & Kota, Rajasthan & 221 & 19-P3 & Indore \\
\hline 179 & SL-637 & PAU, Ludhiana & 222 & JS-2000-20 & Jabalpur \\
\hline 180 & UGM-20075 & Pantnagar & 223 & MRSB-352 & Unknown \\
\hline 181 & PK-1387 & Pantnagar & 224 & NRC 65 & Indore \\
\hline 182 & HiMSO-1598 & Palampur & 225 & PS-1370 & Pantnagar \\
\hline 183 & PK-1347 & Pantnagar & 226 & RKS-15 & Kota, Rajasthan \\
\hline 184 & TS-148-22 & BARC & 227 & TS-39 & BARC \\
\hline 185 & MACS-757 & Pune & 228 & SL-518 & PAU, Ludhiana \\
\hline 186 & MACS-869 & Pune & 229 & HiMSO-1602 & Palampur \\
\hline 187 & TS-3 & BARC & 230 & HiMSO-24 & Palampur \\
\hline 188 & SL-633 & PAU, Ludhiana & 231 & PS-1374 & Pantnagar \\
\hline 189 & DS-200 & DS-Delhi & 232 & PS-1394 & Pantnagar \\
\hline 190 & DS-960 & DS-Delhi & 233 & JS (SH)-40 & Sehore, MP \\
\hline 191 & PS-9813 & Pantnagar & 234 & $\mathrm{JS}(\mathrm{SH})-40$ & Sehore, MP \\
\hline 192 & PS-9816 & Pantnagar & 235 & MRSB-345 & Unknown \\
\hline 193 & PS-9817 & Pantnagar & 236 & VSL 61 & PAU, Ludhiana \\
\hline 194 & PS-9819 & Pantnagar & 237 & SL 679 & PAU, Ludhiana \\
\hline 195 & PS-9820 & Pantnagar & 238 & SL-710 & PAU, Ludhiana \\
\hline 196 & PS-9822 & Pantnagar & 239 & PK-1347 & Pantnagar \\
\hline 197 & DS-9814 & DS-Delhi & 240 & SL 637 & PAU, Ludhiana \\
\hline 198 & DS-9721 & DS-Delhi & 241 & PS-633 & Pantnagar \\
\hline 199 & HiMSO-1563 & Palampur & 242 & PS-1368 & Pantnagar \\
\hline 200 & HiMSO-1587 & Palampur & 243 & SL-638 & PAU, Ludhiana \\
\hline 201 & JS-94-67 & Jabalpur & 244 & AMS-99-22 & Amravati \\
\hline 202 & JS-(SH)-95-26 & Sehore & 245 & SL-751 & PAU, Ludhiana \\
\hline 203 & KB-222 & Kasbe-Digraj & 246 & PKS-25 & Pantnagar \\
\hline 204 & Lee & USA & 247 & AMS-353 & Amravati \\
\hline 205 & MAUS-62-2 & Parbhani & 248 & SL-717 & PAU, Ludhiana \\
\hline 206 & MAUS-64-1 & Parbhani & 249 & PKS-34 & Pantnagar \\
\hline 207 & MAUS-31 & Parbhani & 250 & AMS-47 & Amravati \\
\hline 208 & NRC 45 & Indore & 251 & PS-1403 & Pantnagar \\
\hline 209 & NRC 47 & Indore & 252 & MAUS-231 & Parbhani \\
\hline 210 & NRC 51 & Indore & 253 & NRC-72 & Indore \\
\hline 211 & NRC 53 & Indore & 254 & MAUS-49-1-2 & Parbhani \\
\hline 212 & PK-1024 & Pantnagar & 255 & PS-1410 & Pantnagar \\
\hline 213 & PK-1041 & Pantnagar & 256 & TANUS-55 & TNAU \\
\hline 214 & PK-1060 & Pantnagar & 257 & VLS-65 & VPKS, Almora \\
\hline 215 & PK-1081 & Pantnagar & 258 & KDS-256 & Kasbe-Digraj, \\
\hline
\end{tabular}


Table.1 List of soybean genotypes and their origin used for screening against terminal drought using KI during Kharif-2014

\begin{tabular}{|c|c|c|c|c|c|}
\hline S. No & Genotype & origin & S. No & Genotype & origin \\
\hline 259 & PS-1420 & Pantnagar & 302 & NRC-89 & Indore \\
\hline 260 & PS-1415 & Pantnagar & 303 & DSb-19 & Dharwad \\
\hline 261 & PKS-36 & Pantnagar & 304 & JS-20-38 & Jabalpur \\
\hline 262 & SL-738 & PAU, Ludhiana & 305 & KBS-2011 & Kasbe-Digraj \\
\hline 263 & MACS-1037 & Pune & 306 & VLS-201 & VPKS, Almora \\
\hline 264 & NRC-24 & Indore & 307 & KBS-2010 & Kasbe-Digraj \\
\hline 265 & DS-2309 & DS-Delhi & 308 & DS-2706 & DS-Delhi \\
\hline 266 & SL-747 & PAU, Ludhiana & 309 & PS-1499 & Pantnagar \\
\hline 267 & VLS-66 & VPKS, Almora & 310 & RKS-66 & Kota, Rajasthan \\
\hline 268 & Dsb-9 & Dharwad & 311 & NRC-89 & Indore \\
\hline 269 & AMS-99-33 & Amravati & 312 & VLS 81 & Almora \\
\hline 270 & Dsb-10 & Dharwad & 313 & AMS-56 & Amravati \\
\hline 271 & PS-1437 & Pantnagar & 314 & JS 2038 & Jabalpur \\
\hline 272 & NSO-111 & MAHYCO & 315 & PS 1503 & Pantnagar \\
\hline 273 & SL-452 & PAU, Ludhiana & 316 & HiMSO 1681 & Palampur \\
\hline 274 & MACS-1126 & Pune & 317 & MACS 1364 & Pune \\
\hline 275 & NSO-78 & MAHYCO & 318 & KDS 701 & Kasbe-Digraj \\
\hline 276 & NRC-78 & Indore & 319 & MAUS 608 & Parbhani \\
\hline 277 & MAUS-295 & Parbhani & 320 & KBS 2011 & Kasbe-Digraj \\
\hline 278 & SL-790 & PAU, Ludhiana & 321 & NRC-94 & Indore \\
\hline 279 & AMS-4-63 & Amravati & 322 & RKS-115 & Kota, Rajasthan \\
\hline 280 & NRC-76 & Indore & 323 & VLS-84 & Almora \\
\hline 281 & PS-1454 & Pantnagar & 324 & NRC-93 & Indore \\
\hline 282 & MACS-1184 & Pune & 325 & MACS-1416 & Pune \\
\hline 283 & PS-1450 & Pantnagar & 326 & PS-1521 & Pantnagar \\
\hline 284 & DS-2614 & IARI, Delhi & 327 & NRC-91 & Indore \\
\hline 285 & JS-20-05 & Jabalpur & 328 & MACS-1394 & Pune \\
\hline 286 & JS-20-19 & Jabalpur & & Checks & \\
\hline 287 & JS(SH)-2 & Sehore & 329 & Pusa 9712 & IARI, Delhi \\
\hline 288 & Dsb-15 & Dharwad & 330 & SL 688 & PAU, Ludhiana \\
\hline 289 & PS-1466 & Pantnagar & 331 & PS 1347 & Pantnagar \\
\hline 290 & MACS-1259 & Pune & 332 & PS 1092 & Pantnagar \\
\hline 291 & JS-20-21 & Jabalpur & 333 & Bragg & USA \\
\hline 292 & PS-1476 & Pantnagar & & & \\
\hline 293 & DS-12-5 & DS-Delhi & & & \\
\hline 294 & PS-1480 & Pantnagar & & & \\
\hline 295 & SL-871 & PAU, Ludhiana & & & \\
\hline 296 & DS-27-11 & DS-Delhi & & & \\
\hline 297 & MACS 1340 & Pune & & & \\
\hline 298 & JS 20-35 & Jabalpur & & & \\
\hline 299 & JS(SH)-93-37 & Sehore & & & \\
\hline 300 & KBS-2010 & Kasbe-Digraj & & & \\
\hline 301 & SL-900 & PAU, Ludhiana & & & \\
\hline
\end{tabular}


Table.1a List of soybean genotypes used for screening against terminal drought using KI during Kharif-2015

\begin{tabular}{|c|l|c|l|}
\hline S. No & \multicolumn{1}{|c|}{ Genotype } & S. No & \multicolumn{1}{|c|}{ Genotype } \\
\hline $\mathbf{1}$ & DS-9802 & 24 & VSL-61 \\
\hline $\mathbf{2}$ & EC-97351 & 25 & SL-679 \\
\hline $\mathbf{3}$ & EC-105790 & 26 & PK-1347 \\
\hline $\mathbf{4}$ & G-2130 & 27 & PS-1368 \\
\hline $\mathbf{5}$ & PK-1169 & 28 & SL-751 \\
\hline $\mathbf{6}$ & PK-1180 & 29 & PKS-25 \\
\hline $\mathbf{7}$ & PK-1240 & 30 & PKS-34 \\
\hline $\mathbf{8}$ & PK-1243 & 31 & AMS-47 \\
\hline $\mathbf{9}$ & SL-46 & 32 & KDS-256 \\
\hline $\mathbf{1 0}$ & SL-432 & 33 & PS-1420 \\
\hline $\mathbf{1 1}$ & TGX1828-4E & 34 & PS-1415 \\
\hline $\mathbf{1 2}$ & TGX1835-3E & 35 & DS-2309 \\
\hline $\mathbf{1 3}$ & UPSL-291 & 36 & MACS-1126 \\
\hline $\mathbf{1 4}$ & UPSL-298 & 37 & NRC-78 \\
\hline $\mathbf{1 5}$ & EC-471979 & 38 & KBS-2010 \\
\hline $\mathbf{1 6}$ & SL-633 & 39 & AMS-56 \\
\hline $\mathbf{1 7}$ & HiMSO-1563 & 40 & PS-9818 \\
\hline $\mathbf{1 8}$ & HiMSO-1587 & & \\
\hline $\mathbf{1 9}$ & KB-222 & 1 & Pusa-9712 \\
\hline $\mathbf{2 0}$ & PK-1024 & 2 & SL-688 \\
\hline $\mathbf{2 1}$ & JS-2000-20 & 3 & PS-1347 \\
\hline $\mathbf{2 2}$ & RKS-15 & 4 & PS-1092 \\
\hline $\mathbf{2 3}$ & TS-39 & 5 & Bragg \\
\hline
\end{tabular}


Table.2a Analysis of variance for growth and yield parameters of germplasm lines grown under control conditions Kharif-2014

\begin{tabular}{|c|c|c|c|c|c|c|c|c|c|}
\hline Source of variation & df & $\begin{array}{c}\text { Days to } \\
50 \% \\
\text { flowering }\end{array}$ & $\begin{array}{l}\text { Days to full } \\
\text { maturity }\end{array}$ & $\begin{array}{l}\text { 100-seed } \\
\text { weight }\end{array}$ & $\begin{array}{l}\text { No. of pods } \\
\text { per plant }\end{array}$ & $\begin{array}{c}\text { No. of } \\
\text { seeds per } \\
\text { pod }\end{array}$ & $\begin{array}{c}\text { Plant } \\
\text { height }\end{array}$ & Row yield & $\begin{array}{c}\text { Single } \\
\text { plant yield }\end{array}$ \\
\hline Block & 6 & $\begin{array}{c}5.86^{\mathrm{NS}} \\
(0.6715)\end{array}$ & $\begin{array}{c}18.86^{\mathrm{NS}} \\
(0.068)\end{array}$ & $\begin{array}{c}1.05^{\mathrm{NS}} \\
(0.4536)\end{array}$ & $\begin{array}{c}77.18^{\mathrm{NS}} \\
(0.1635)\end{array}$ & $\begin{array}{c}0.02^{\mathrm{NS}} \\
(0.4481)\end{array}$ & $\begin{array}{c}57.90^{\mathrm{NS}} \\
(0.6581)\end{array}$ & $\begin{array}{c}4983.62^{\mathrm{NS}} \\
(0.0031)\end{array}$ & $\begin{array}{c}3.90^{\mathrm{NS}} \\
(0.1065)\end{array}$ \\
\hline Treatments & 332 & $\begin{array}{l}61.14^{* * *} \\
(<.0001)\end{array}$ & $\begin{array}{l}47.38^{* *} \\
(<.0001)\end{array}$ & $\begin{array}{c}7.00^{* *} \\
(<.0001)\end{array}$ & $\begin{array}{l}807.63^{* *} \\
(<.0001)\end{array}$ & $\begin{array}{c}0.22^{* *} \\
(<.0001)\end{array}$ & $\begin{array}{c}173.38^{*} \\
(0.0175)\end{array}$ & $\begin{array}{c}20047.16^{* *} \\
(<.0001)\end{array}$ & $\begin{array}{l}34.74^{* *} \\
(<.0001)\end{array}$ \\
\hline Tests & 327 & $\begin{array}{l}52.54^{* * *} \\
(<.0001)\end{array}$ & $\begin{array}{l}44.45^{* *} \\
(<.0001)\end{array}$ & $\begin{array}{c}6.59^{* *} \\
(<.0001)\end{array}$ & $\begin{array}{l}773.05^{* *} \\
(<.0001)\end{array}$ & $\begin{array}{c}0.20^{* *} \\
(<.0001)\end{array}$ & $\begin{array}{c}159.36^{*} \\
(0.0303)\end{array}$ & $\begin{array}{c}12664.38^{* *} \\
(<.0001)\end{array}$ & $\begin{array}{l}28.60^{* *} \\
(<.0001)\end{array}$ \\
\hline Among Checks & 4 & $\begin{array}{l}641.1^{* *} \\
(<.0001)\end{array}$ & $\begin{array}{l}233.33^{* *} \\
(<.0001)\end{array}$ & $\begin{array}{c}4.76 \\
(0.0075)\end{array}$ & $\begin{array}{l}1341.23^{* * *} \\
(<.0001)\end{array}$ & $\begin{array}{c}0.020^{\mathrm{NS}} \\
(0.4269)\end{array}$ & $\begin{array}{l}1240.74^{* *} \\
(<.0001)\end{array}$ & $\begin{array}{c}94343.21^{* *} \\
(<.0001)\end{array}$ & $\begin{array}{c}3.84^{\mathrm{NS}} \\
(0.1325)\end{array}$ \\
\hline Test V/s Checks & 1 & $\begin{array}{l}594.05^{\text {** }} \\
(<.0001)\end{array}$ & $\begin{array}{l}271.78^{* * *} \\
(<.0001)\end{array}$ & $\begin{array}{l}154.84^{* * *} \\
(<.0001)\end{array}$ & $\begin{array}{c}10975.14^{* *} \\
(<.0001)\end{array}$ & $\begin{array}{c}5.70^{* *} \\
(<.0001)\end{array}$ & $\begin{array}{c}564.13^{*} \\
(0.0158)\end{array}$ & $\begin{array}{c}2198899.89^{* *} \\
\quad(<.0001)\end{array}$ & $\begin{array}{l}2363.53^{* *} \\
(<.0001)\end{array}$ \\
\hline F.rror & 24 & 870 & 821 & 106 & 45.32 & 002 & 8366 & 1085.16 & 196 \\
\hline CD at 5\% A mono Test & & 943 & 916 & 329 & 2152 & ก 54 & 2924 & 10532 & 447 \\
\hline CD at 5\% Test Vs checks & & 697 & 678 & 2.43 & 1597 & ก 399 & 2164 & 7732 & 3.31 \\
\hline CV $(\%)$ & & 541 & 2.43 & 1199 & 1083 & 6.54 & 12.63 & 1876 & 1577 \\
\hline
\end{tabular}

NS: Non significant; ** highly significant; * significant; values in parenthesis indicate the Probability value $(\operatorname{Pr}>\mathrm{F})$. 
Table.2b Analysis of variance for growth and yield parameters of germplasm lines sprayed with Potassium Iodide (KI) Kharif-2014

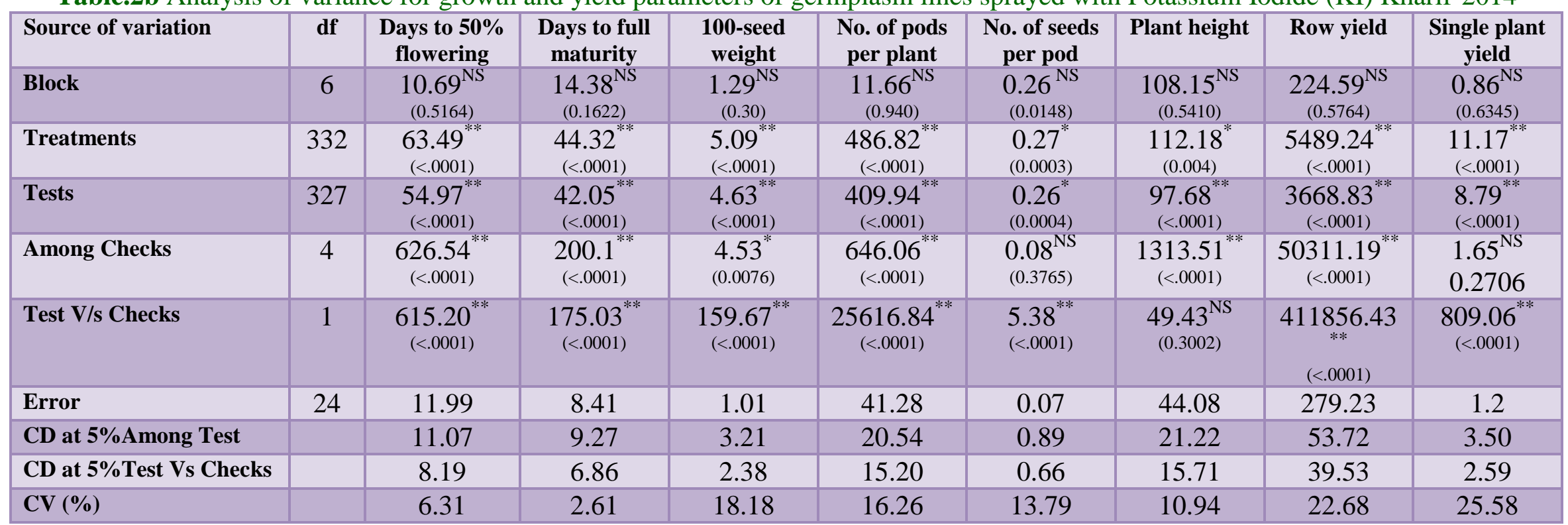

NS: Non significant; ** highly significant; * Significant; Values in parenthesis indicate the Probability value $(\operatorname{Pr}>\mathrm{F})$.

Table.2c Descriptive statistics for quantitative characters of 328 genotypes screened for drought tolerance under control and KI treated conditions

\begin{tabular}{|c|c|c|c|c|c|c|c|c|c|c|c|c|c|c|}
\hline \multirow[b]{2}{*}{ Characters } & \multicolumn{2}{|c|}{ Max. } & \multicolumn{2}{|c|}{ Min. } & \multicolumn{2}{|c|}{ Range } & \multicolumn{2}{|c|}{ Mean } & \multicolumn{2}{|c|}{$\mathrm{CV} \%$} & \multicolumn{2}{|c|}{ SE(d) } & \multicolumn{2}{|c|}{ CD among tests $(0.05)$} \\
\hline & $\mathbf{C}$ & $\mathbf{T}$ & $\mathbf{C}$ & $\mathbf{T}$ & $\mathbf{C}$ & $\mathbf{T}$ & $\mathbf{C}$ & $\mathbf{T}$ & $\mathbf{C}$ & $\mathbf{T}$ & $\mathbf{C}$ & $\mathbf{T}$ & $\mathbf{C}$ & $\mathbf{T}$ \\
\hline Days to $50 \%$ flowering & 68.80 & 68.77 & 36.6 & 34.17 & 32.2 & 34.6 & 54.92 & 55.17 & 5.41 & 6.31 & 0.469 & 0.484 & 9.43 & 11.07 \\
\hline Days to full maturity & 147.4 & 124.74 & 99.94 & 98.34 & 47.46 & 26.4 & 118.16 & 110.04 & 2.43 & 2.61 & 0.398 & 0.388 & 9.16 & 9.27 \\
\hline 100-seed weight & 16.12 & 13.15 & 2.46 & 0.41 & 13.66 & 12.74 & 8.41 & 5.33 & 11.99 & 18.18 & 0.153 & 0.122 & 3.29 & 3.21 \\
\hline No. of pods per plant & 174.48 & 145.11 & 13.39 & 4.6 & 161.09 & 140.5 & 60.86 & 37.1 & 10.83 & 16.26 & 1.724 & 1.225 & 21.52 & 20.54 \\
\hline No. of seeds per pod & 3.32 & 2.97 & 1.97 & 0.88 & 1.35 & 2.08 & 2.56 & 1.99 & 6.54 & 13.79 & 0.027 & 0.029 & 0.54 & 0.89 \\
\hline Plant height & 110.06 & 96.83 & 32.44 & 32.4 & 77.62 & 64.43 & 72.96 & 60.66 & 12.63 & 10.94 & 0.705 & 0.554 & 29.24 & 21.22 \\
\hline Row yield & 636.57 & 351.37 & 1.47 & 0.17 & 635.09 & 351.2 & 158.45 & 62.38 & 18.76 & 22.68 & 7.292 & 3.400 & 105.32 & 53.72 \\
\hline Single plant yield & 37.37 & 15.02 & 0.22 & 0.11 & 37.15 & 15.02 & 8.2 & 3.86 & 15.77 & 25.58 & 0.353 & 0.175 & 4.47 & 3.5 \\
\hline
\end{tabular}

C: control, T: treatment 
Table.3a Analysis of variance for growth and yield parameters of germplasm lines grown under control conditions Kharif-2015

\begin{tabular}{|c|c|c|c|c|c|c|c|c|c|}
\hline Source of variation & df & $\begin{array}{c}\text { Days to } \\
50 \% \\
\text { flowering }\end{array}$ & $\begin{array}{l}\text { Days to full } \\
\text { maturity }\end{array}$ & $\begin{array}{l}\text { 100-seed } \\
\text { weight }\end{array}$ & $\begin{array}{l}\text { No. of pods } \\
\text { per plant }\end{array}$ & $\begin{array}{l}\text { No. of } \\
\text { seeds per } \\
\text { pod }\end{array}$ & $\begin{array}{l}\text { Plant } \\
\text { height }\end{array}$ & Row yield & $\begin{array}{c}\text { Single } \\
\text { plant yield }\end{array}$ \\
\hline Block & 1 & $\begin{array}{l}1.60^{\mathrm{NS}} \\
(0.662)\end{array}$ & $\begin{array}{l}1.877^{\mathrm{NS}} \\
(0.350)\end{array}$ & $\begin{array}{l}0.215 \mathrm{NS} \\
(0.0018)\end{array}$ & $\begin{array}{c}187.06^{\mathrm{NS}} \\
(0.058)\end{array}$ & $\begin{array}{l}0.072^{\mathrm{NS}} \\
(0.467)\end{array}$ & $\begin{array}{l}7.91^{\mathrm{NS}} \\
(0.135)\end{array}$ & $\begin{array}{l}0.008^{N S} \\
(0.921)\end{array}$ & $\begin{array}{l}3.66^{\mathrm{NS}} \\
(0.126)\end{array}$ \\
\hline Treatments & 44 & $\begin{array}{l}95.26^{* *} \\
(<.0001)\end{array}$ & $\begin{array}{l}131.64^{* *} \\
(<.0001)\end{array}$ & $\begin{array}{c}2.57^{* *} \\
(<.0006)\end{array}$ & $\begin{array}{l}292.11^{* *} \\
(<.0001)\end{array}$ & $\begin{array}{l}0.2082^{\mathrm{NS}} \\
(<.0736)\end{array}$ & $\begin{array}{l}188.24^{* *} \\
(<.0001)\end{array}$ & $\begin{array}{c}11668.13 * * \\
(<.0001)\end{array}$ & $\begin{array}{l}12.86^{* *} \\
(<.0001)\end{array}$ \\
\hline Error & 44 & 8.25 & 2.10 & 0.94 & 49.61 & 0.134 & 3.41 & 105.55 & 1.50 \\
\hline CD at $5 \%$ & & 5.79 & 2.92 & 1.95 & 14.19 & NS & 3.72 & 20.70 & 2.47 \\
\hline CV $(\%)$ & & 5.58 & 1.33 & 12.36 & 17.02 & 47.62 & 3.69 & 9.15 & 16.79 \\
\hline
\end{tabular}

Table.3b Analysis of variance for growth and yield parameters of germplasm lines grown under KI treated conditions Kharif-2015

\begin{tabular}{|c|c|c|c|c|c|c|c|c|c|}
\hline Source of variation & df & $\begin{array}{c}\text { Days to } \\
50 \% \\
\text { flowering }\end{array}$ & $\begin{array}{l}\text { Days to full } \\
\text { maturity }\end{array}$ & $\begin{array}{c}\text { 100-seed } \\
\text { weight }\end{array}$ & $\begin{array}{c}\text { No. of pods } \\
\text { per plant }\end{array}$ & $\begin{array}{c}\text { No. of } \\
\text { seeds per } \\
\text { pod }\end{array}$ & $\begin{array}{c}\text { Plant } \\
\text { height }\end{array}$ & Row yield & $\begin{array}{c}\text { Single } \\
\text { plant yield }\end{array}$ \\
\hline Block & 1 & $\begin{array}{l}30.04^{\mathrm{NS}} \\
(0.306)\end{array}$ & $\begin{array}{l}6.94^{\mathrm{NS}} \\
(0.691)\end{array}$ & $\begin{array}{l}0.105^{\mathrm{NS}} \\
(0.6108)\end{array}$ & $\begin{array}{l}4.32^{N S} \\
(0.712)\end{array}$ & $\begin{array}{c}0.0393^{\mathrm{NS}} \\
(0.2737)\end{array}$ & $\begin{array}{l}153.83^{\mathrm{NS}} \\
(0.0848)\end{array}$ & $\begin{array}{c}19.40463^{\mathrm{NS}} \\
(0.7116)\end{array}$ & $\begin{array}{c}0.0003^{\mathrm{NS}} \\
(0.982)\end{array}$ \\
\hline Treatments & 44 & $\begin{array}{l}98.29^{* * *} \\
(<.0001)\end{array}$ & $\begin{array}{l}140.45^{* *} \\
(<.0001)\end{array}$ & $\begin{array}{c}2.92^{* *} \\
(<.0001)\end{array}$ & $\begin{array}{l}126.74^{* *} \\
(<.0001)\end{array}$ & $\begin{array}{l}0.0913^{* *} \\
(<.0001)\end{array}$ & $\begin{array}{l}85.83^{* *} \\
(0.0356)\end{array}$ & $\begin{array}{l}4414.41^{* *} \\
(<.0001)\end{array}$ & $\begin{array}{l}6.233^{* *} \\
(<.0001)\end{array}$ \\
\hline Error & 44 & 28.08 & 43.53 & 0.399 & 31.47 & 0.032 & 49.49 & 43.11 & 0.659 \\
\hline CD at $5 \%$ & & 10.68 & 13.29 & 1.27 & 11.30 & 0.36 & 5.18 & 13.23 & 1.63 \\
\hline $\mathrm{CV}(\%)$ & & 10.32 & 10.67 & 12.72 & 24.55 & 8.08 & 5.21 & 9.15 & 20.00 \\
\hline
\end{tabular}


Table.4 Shannon-Weaver diversity indices for qualitative traits of the 328 germplasm accessions

\begin{tabular}{|c|l|c|}
\hline Sl. No. & Character & $\mathbf{H}^{\mathbf{I}}$ \\
\hline $\mathbf{1}$ & Growth habit & 0.69 \\
\hline $\mathbf{2}$ & Leaf shape & 0.25 \\
\hline $\mathbf{3}$ & Flower colour & 0.67 \\
\hline $\mathbf{4}$ & Pod colour & 0.21 \\
\hline $\mathbf{5}$ & Pod pubescence & 0.13 \\
\hline $\mathbf{6}$ & Pubescence colour & 0.26 \\
\hline $\mathbf{7}$ & Seed Shape & 0.69 \\
\hline $\mathbf{8}$ & Seed colour & 1.2 \\
\hline $\mathbf{9}$ & Seed lustre & 0.69 \\
\hline $\mathbf{1 0}$ & Hilum colour & 0.93 \\
\hline & Average & 0.57 \\
\hline
\end{tabular}

Table.4a Estimation of Principal component, Eigen value, proportional and cumulative percent variation for control block

\begin{tabular}{|c|c|c|c|}
\hline Component & Eigen Values & Variability \% & Cumulative $\%$ \\
\hline $\mathbf{P}_{\mathbf{1}}$ & 4.0985 & 51.2307 & 51.2307 \\
\hline $\mathbf{P}_{\mathbf{2}}$ & 1.0853 & 13.5662 & 64.7969 \\
\hline $\mathbf{P}_{\mathbf{3}}$ & 0.7750 & 9.6879 & 74.4848 \\
\hline $\mathbf{P}_{\mathbf{4}}$ & 0.6151 & 7.6885 & 82.1733 \\
\hline $\mathbf{P}_{\mathbf{5}}$ & 0.5142 & 6.4275 & 88.6008 \\
\hline $\mathbf{P}_{\mathbf{6}}$ & 0.3798 & 4.7471 & 93.3479 \\
\hline $\mathbf{P}_{\mathbf{7}}$ & 0.2868 & 3.5847 & 96.9325 \\
\hline $\mathbf{P}_{\mathbf{8}}$ & 0.2454 & 3.0675 & 100.0000 \\
\hline
\end{tabular}

Table.4b Estimation of Principal component, Eigen value, proportional and cumulative percent variation for Treatment block

\begin{tabular}{|c|c|c|c|}
\hline Component & Eigen Values & Variability \% & Cumulative \% \\
\hline $\mathbf{P}_{\mathbf{1}}$ & 3.7417 & 46.7711 & 46.7711 \\
\hline $\mathbf{P}_{\mathbf{2}}$ & 1.1953 & 14.9408 & 61.7119 \\
\hline $\mathbf{P}_{\mathbf{3}}$ & 0.7926 & 9.9073 & 71.6193 \\
\hline $\mathbf{P}_{\mathbf{4}}$ & 0.7169 & 8.9615 & 80.5808 \\
\hline $\mathbf{P}_{\mathbf{5}}$ & 0.5665 & 7.0812 & 87.6620 \\
\hline $\mathbf{P}_{\mathbf{6}}$ & 0.4199 & 5.2489 & 92.9109 \\
\hline $\mathbf{P}_{\mathbf{7}}$ & 0.2989 & 3.7357 & 96.6466 \\
\hline $\mathbf{P}_{\mathbf{8}}$ & 0.2683 & 3.3534 & 100.0000 \\
\hline
\end{tabular}


Table.5a Estimation of percent contribution of traits for principal component in control block

\begin{tabular}{|l|c|c|c|c|c|c|c|c|}
\hline \multicolumn{1}{|c|}{ Characters } & $\mathbf{P}_{\mathbf{1}}$ & $\mathbf{P}_{\mathbf{2}}$ & $\mathbf{P}_{\mathbf{3}}$ & $\mathbf{P}_{\mathbf{4}}$ & $\mathbf{P}_{\mathbf{5}}$ & $\mathbf{P}_{\mathbf{6}}$ & $\mathbf{P}_{\mathbf{7}}$ & $\mathbf{P}_{\mathbf{8}}$ \\
\hline Days to 50\% & 14.06 & 9.52 & 2.65 & 26.09 & 2.06 & 0.31 & 40.04 & 5.26 \\
\hline Days to full & 15.39 & 1.93 & 7.83 & 22.96 & 0.00 & 1.70 & 40.06 & 10.12 \\
\hline 100-seed weight & 13.35 & 1.93 & 2.46 & 0.00 & 77.96 & 1.17 & 0.02 & 3.11 \\
\hline No. of pods per & 15.58 & 1.09 & 14.52 & 1.56 & 7.79 & 29.77 & 3.43 & 26.27 \\
\hline No. of seeds per & 8.11 & 3.57 & 60.42 & 23.89 & 0.00 & 2.40 & 1.09 & 0.51 \\
\hline Plant height & 0.36 & 80.12 & 0.27 & 16.95 & 1.23 & 0.02 & 1.05 & 0.00 \\
\hline Row yield & 15.93 & 0.87 & 1.41 & 3.61 & 10.23 & 62.26 & 3.90 & 1.78 \\
\hline Single plant yield & 17.22 & 0.96 & 10.43 & 4.93 & 0.72 & 2.38 & 10.40 & 52.95 \\
\hline
\end{tabular}

Table.5b Estimation of percent contribution of traits for principal component in treatment block

\begin{tabular}{|l|c|c|c|c|c|c|c|c|}
\hline \multicolumn{1}{|c|}{ Characters } & $\mathbf{P}_{\mathbf{1}}$ & $\mathbf{P}_{\mathbf{2}}$ & $\mathbf{P}_{\mathbf{3}}$ & $\mathbf{P}_{\mathbf{4}}$ & $\mathbf{P}_{\mathbf{5}}$ & $\mathbf{P}_{\mathbf{6}}$ & $\mathbf{P}_{\mathbf{7}}$ & $\mathbf{P}_{\mathbf{8}}$ \\
\hline Days to 50\% & 12.87 & 18.08 & 0.00 & 13.90 & 13.22 & 3.63 & 32.26 & 6.04 \\
\hline Days to full & 17.17 & 3.68 & 1.20 & 2.56 & 22.88 & 1.03 & 44.28 & 7.20 \\
\hline 100-seed weight & 10.14 & 2.05 & 2.17 & 68.43 & 13.64 & 0.36 & 3.09 & 0.13 \\
\hline No. of pods per & 18.29 & 1.31 & 1.37 & 0.47 & 14.85 & 19.94 & 2.12 & 41.66 \\
\hline No. of seeds per & 6.42 & 8.87 & 80.53 & 0.05 & 0.69 & 0.96 & 2.00 & 0.49 \\
\hline Plant height & 0.06 & 64.08 & 4.75 & 13.69 & 16.20 & 0.60 & 0.51 & 0.12 \\
\hline Row yield & 16.30 & 0.59 & 7.43 & 0.83 & 5.42 & 66.00 & 3.24 & 0.18 \\
\hline Single plant yield & 18.75 & 1.34 & 2.55 & 0.07 & 13.10 & 7.48 & 12.50 & 44.20 \\
\hline
\end{tabular}

Table.5c Loadings of principle component for PC1 and PC2

\begin{tabular}{|l|c|c|c|c|}
\hline \multicolumn{1}{|c|}{ Loadings } & \multicolumn{2}{c|}{ Control } & \multicolumn{2}{c|}{ Treatment } \\
\hline & $\mathrm{P}_{1}$ & $\mathrm{P}_{2}$ & $\mathrm{P}_{1}$ & $\mathrm{P}_{2}$ \\
\hline Days to 50\% & -0.759 & 0.321 & -0.694 & 0.465 \\
\hline Days to full maturity & -0.794 & 0.145 & -0.802 & 0.210 \\
\hline 100-seed weight & 0.740 & 0.145 & 0.616 & 0.157 \\
\hline No. of pods per plant & 0.799 & 0.109 & 0.827 & 0.125 \\
\hline No. of seeds per pod & 0.577 & 0.197 & 0.490 & 0.326 \\
\hline Plant height & -0.121 & 0.933 & -0.046 & 0.875 \\
\hline Row yield & 0.808 & 0.097 & 0.781 & 0.084 \\
\hline Single plant yield & 0.840 & 0.102 & 0.838 & 0.127 \\
\hline
\end{tabular}


Table.6 Seed yield, seeds per plant, 100-seed weight and pods per plant of 328 soybean genotypes evaluated for terminal drought tolerance during Kharif-2014

\begin{tabular}{|l|c|c|c|c|c|c|c|c|}
\hline \multirow{2}{*}{ Characters } & \multicolumn{3}{|c|}{ Control } & \multicolumn{3}{c|}{ Treatment } & \multicolumn{2}{c|}{$\begin{array}{c}\text { Percent reduction due to KI } \\
\text { spray }\end{array}$} \\
\cline { 2 - 10 } & Mean & Range & CV\% & Mean & Range & CV\% & Mean & $1.94-97.44$ \\
\hline Seed yield (g/row) & 175.57 & $6.00-634.00$ & 18.76 & 67.88 & $2.00-308.00$ & 22.68 & 61.34 & $0.54-95.01$ \\
\hline Seed yield (g/plant) & 8.86 & $1.60-36.13$ & 15.77 & 4.26 & $0-15.18$ & 25.58 & 51.92 & $0.62-96.67$ \\
\hline 100-seed weight(g) & 8.59 & $2.50-16.20$ & 11.99 & 5.72 & $0.35-12.56$ & 18.18 & 33.41 & 34.72 \\
\hline No. of pods/plant & 62.10 & $11.00-180.00$ & 10.83 & 40.54 & $4.00-120.66$ & 16.26 & $0.78-87.34$ \\
\hline
\end{tabular}

Table.7 classification of germplasm lines based on the effect of KI spray on row yield and 100-seed weight under control and KI treated conditions during Kharif-2014

\begin{tabular}{|c|c|c|c|c|c|c|}
\hline \multirow{2}{*}{$\begin{array}{c}\text { Sl } \\
\text { No. }\end{array}$} & \multirow[t]{2}{*}{ Group } & \multirow[t]{2}{*}{ Germplasms } & \multicolumn{2}{|c|}{ Row yield $(\mathrm{g})$} & \multicolumn{2}{|c|}{ 100-seed weight $(\mathrm{g})$} \\
\hline & & & Control & KI treated & Control & KI treated \\
\hline \multirow[t]{4}{*}{1} & \multirow[t]{4}{*}{ Tolerant $(0-20 \%)$} & TGX1835-3E & 216.17 & $197.28(8.74 \%)$ & 10.54 & $9.72(7.76 \%)$ \\
\hline & & VSL-61 & 246.77 & $218.84(11.32 \%)$ & 9.06 & $8.82(2.65 \%)$ \\
\hline & & EC-105790 & 248.37 & $223.74(9.92 \%)$ & 10.33 & $9.24(10.53 \%)$ \\
\hline & & PK-1243 & 262.31 & $228.61(12.85 \%)$ & 9.09 & $7.58(16.59 \%)$ \\
\hline \multirow[t]{4}{*}{2} & \multirow[t]{4}{*}{ Moderately tolerant $(20.1-45 \%)$} & G-2130 & 90.17 & $53.47(40.70 \%)$ & 12.48 & $7.34(41.17 \%)$ \\
\hline & & DS-9802 & 122.17 & $83.74(31.70 \%)$ & 9.28 & $6.42(30.80 \%)$ \\
\hline & & SL-633 & 176.32 & $120.42(31.70 \%)$ & 10.69 & $6.71(37.21 \%)$ \\
\hline & & PKS-25 & 216.20 & $146.27(32.35 \%)$ & 11.37 & $8.11(28.65 \%)$ \\
\hline \multirow[t]{4}{*}{3} & \multirow[t]{4}{*}{ Susceptible $(45.1-85 \%)$} & PK-1024 & 76.21 & $28.92(62.04 \%)$ & 12.97 & $6.25(51.84 \%)$ \\
\hline & & PK-1240 & 86.34 & $43.38(49.76 \%)$ & 7.79 & $2.35(69.83 \%)$ \\
\hline & & DS-2309 & 64.57 & $29.25(54.70 \%)$ & 12.86 & $4.01(68.85 \%)$ \\
\hline & & UPSL-291 & 81.54 & $33.81(63.90 \%)$ & 8.78 & $3.07(65.08 \%)$ \\
\hline
\end{tabular}


Table.8 Seed yield, seeds per plant, 100-seed weight and pods per plant of four identified soybean genotypes evaluated for terminal drought tolerance using KI Kharif-2015

\begin{tabular}{|c|l|c|c|c|c|c|c|c|c|}
\hline SI No. & \multicolumn{1}{|c|}{ Characters } & \multicolumn{2}{|c|}{ TGX1835-3E } & \multicolumn{2}{|c|}{ VSL-69 } & \multicolumn{2}{|c|}{ EC-105780 } & KK-1243 \\
\cline { 3 - 10 } & & Control & KI & Control & KI & Control & KI & Control & KI \\
\hline $\mathbf{1}$ & Seed yield (g/row) & 101.64 & $86.65(14.75 \%)$ & 108.45 & $93.46(13.83 \%)$ & 98.78 & $90.31(8.57 \%)$ & 109.73 & $91.96(16.19 \%)$ \\
\hline $\mathbf{2}$ & Seed yield (g/plant) & 8.80 & $7.08(19.59 \%)$ & 7.78 & $6.34(18.49 \%)$ & 9.13 & $7.68(15.79 \%)$ & 10.47 & $8.46(19.22 \%)$ \\
\hline $\mathbf{3}$ & 100-seed weight(g) & 7.45 & $7.10(4.70 \%)$ & 5.20 & $4.90(5.77 \%)$ & 7.40 & $6.75(8.78 \%)$ & 6.40 & $5.78(9.69 \%)$ \\
\hline $\mathbf{4}$ & No. of pods/plant & 65.25 & $53.68(17.73 \%)$ & 61.10 & $51.37(15.92 \%)$ & 50.92 & $40.95(19.57 \%)$ & 51.70 & $42.72(17.38 \%)$ \\
\hline
\end{tabular}

$\mathrm{KI}=$ potassium iodide 
Fig.1a Biplot of 328 soybean germplasm lines on PC1 and PC2 under controlled conditions Kharif-2014

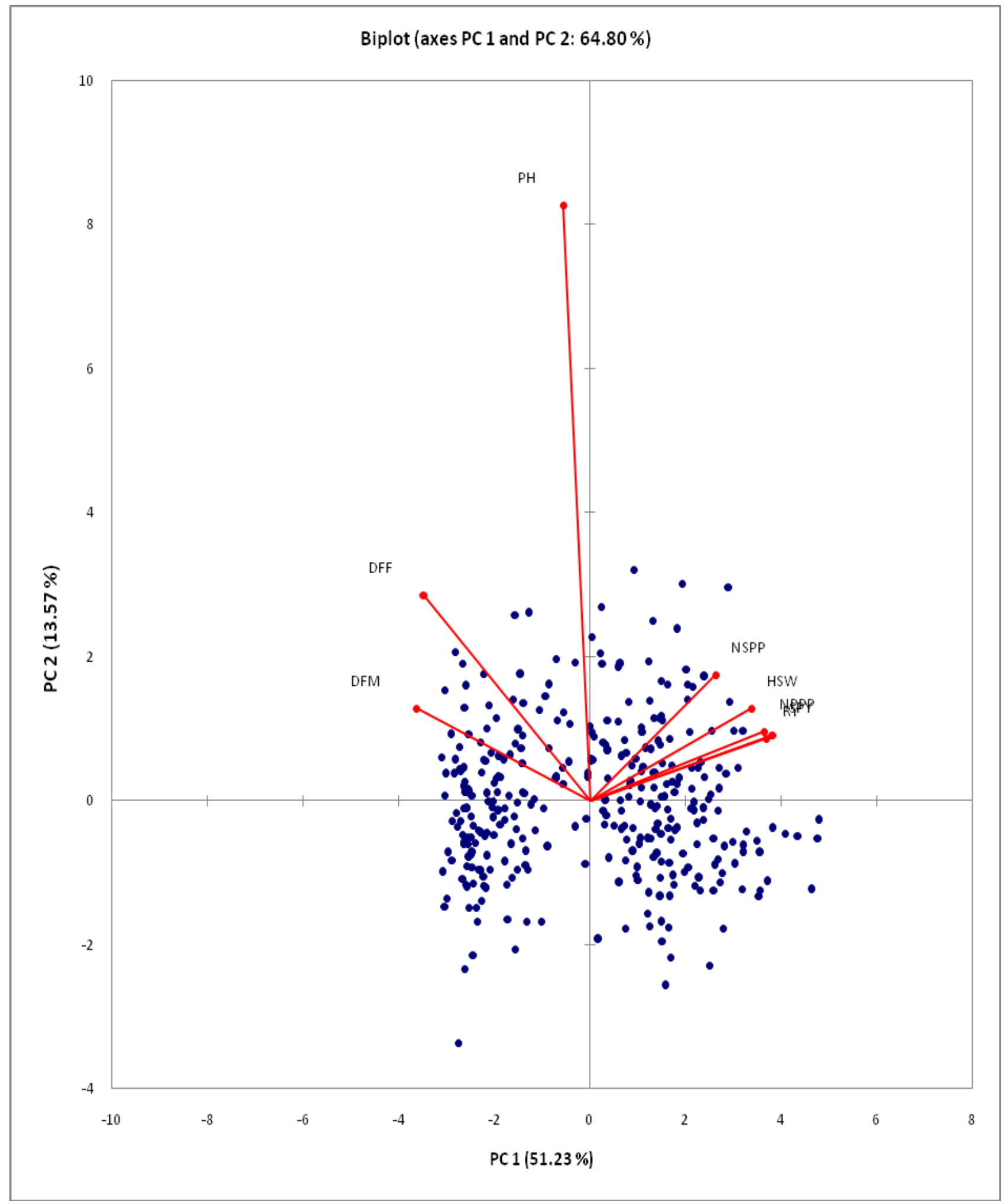


Fig.1b Biplot of 328 soybean germplasm lines on PC1 and PC2 under treated conditions Kharif2014

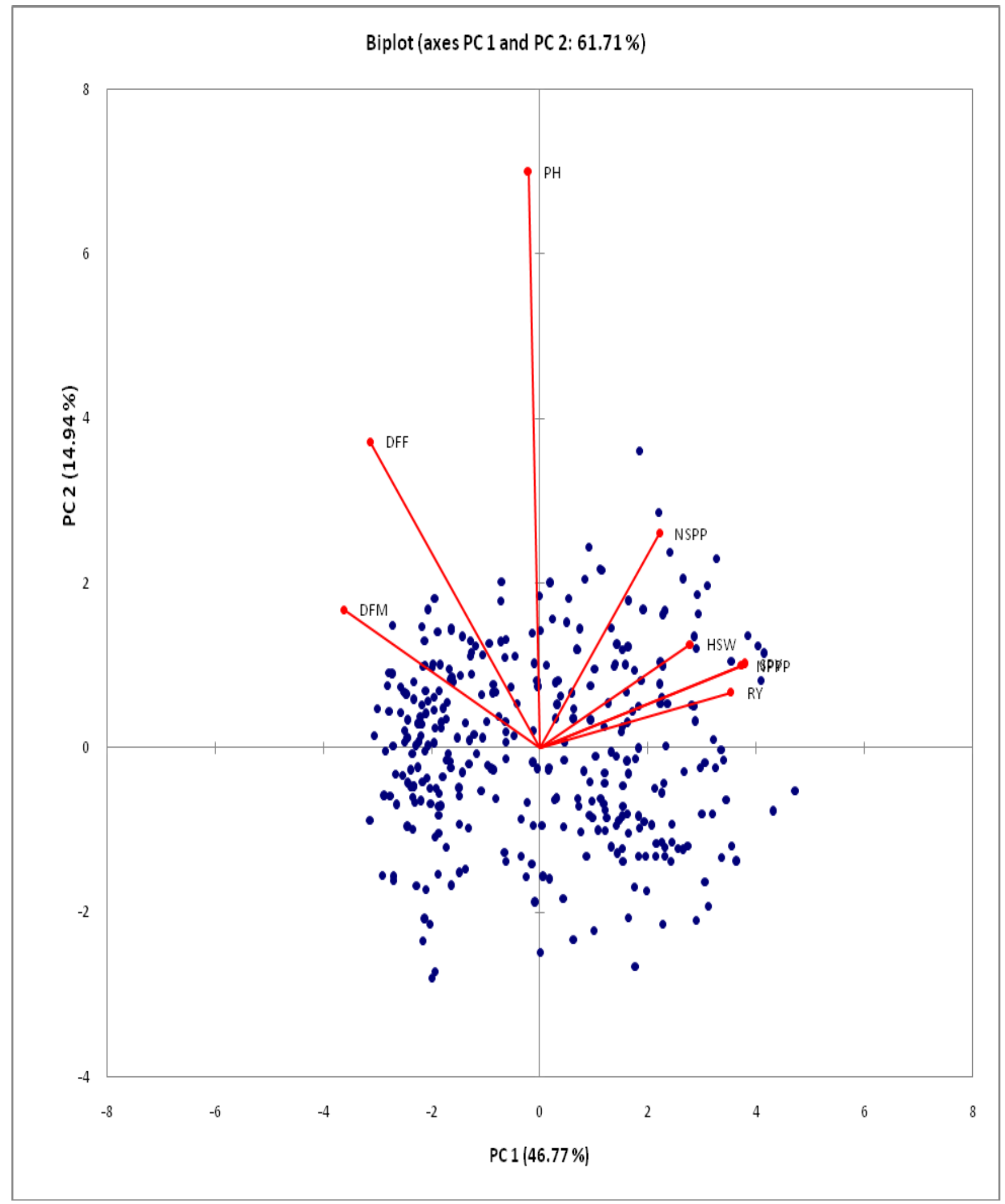


In conclusion, drought is one of the most important factors responsible for the reduction of more than $50 \%$ seed yield in soybean. The occurrence of drought at terminal stage especially at seed filling to seed development in soybean is reported to cause severe yield loss. Drought tolerant genotypes have an inherent ability to increase source to sink mobilization of photosynthates under terminal drought conditions. KI spray at $\mathrm{R}_{5}$ stage would imitate the terminal drought conditions and which is extensively tested in cereals and pulses as a defoliant and also mimics near drought like conditions under field conditions. Under KI treated conditions, the genotypes are placed well apart on a Biplot analysis and thus most lines have diverse expression towards KI spray, which shows that the lines were very divers in nature. In the present study, potassium iodide was applied at $R_{5}$ (seed filling) stage on a 328 germplasm lines to screen for terminal drought tolerance. Four genotypes (TGX1835-3E, VSL-69, EC105780 and PK-1243) were identified as relatively tolerant as they showed least reduction for hundred seed weight and seed yield under KI induced drought over control. The identified genotypes were re-tested under similar conditions in next season. Based on two years testing, genotypes showing consistent behavior for drought tolerance were identified. Further studies by the authors will focus the molecular and physiological mechanisms involved in the drought tolerance in these lines for better utilization in breeding programmes.

\section{Acknowledgments}

The authors are highly thankful to Director, ICAR-IARI, New Delhi for providing facilities and support. The authors acknowledge the support of The Head, Genetics Division, ICAR-IARI New Delhi. The first author is highly thankful to Director, ICAR-SBI, Coimbatore for allowing an InService PhD at ICAR-IARI, New Delhi.

\section{References}

Ashraf M, Arfan M, Ahmad A (2003) Evaluation of the usefulness of senescing agent potassium iodide for assessing inter-cultivar variation for drought tolerance in pearl millet (Pennisetum glaucum (L.) R. Br). Aus J Exp Agric 43:1337-1343

Bhatia VS, Jumrani K, Pandey GP (2014) Evaluation of the usefulness of senescing agent potassium iodide (KI) as a screening tool for tolerance to terminal drought in soybean. Plant Knowledge J 3(1):23-30

Birt DF, Hendrick S, Alekel DL (2004) Soybean and the prevention of chronic human disease in Soybeans: Improvement, Production, and Uses. Agronomy Monographs $3^{\text {rd }}$ ed. No. 16, HR. Boerma and J E. Specht eds. ASACSSA-SSSA, Madison, WI, USA. 1047-1117

Blum A, Mayer J, Gozlan G (1983a) Chemical desiccation of wheat plants as a simulator of post anthesis stress II. Relations with drought stress. Field Crop Res. 6:149-155

Bouslama M, Schapaugh Jr WT (1984) Stress tolerance in Soybeans I. Evaluation of three screening techniques for heat and drought tolerance. Crop Sci 24:932-937

Boyer JS (1982) Plant productivity and environment. Sci. 218:443-448

Brar GS, Kar S, Singh NT (1990) Photosynthetic response of wheat to soil water deficits in the tropics. J Agron Crop Sci 164: 343-348.

Bray EA, Bailey-Serres J, Weretilnyk E (2000) Responses to abiotic stresses in: Biochemistry and molecular biology of plants, Gruissem W, Buchannan B, Jones R. eds. American S. Plant Physiol 1158-1249

Brevedan RE, Egli DB (2003) Short periods of water stress during seed filling, leaf 
senescence, and yield of soybean. Crop Sci 43:2083-2088

Burton GW (1952) Quantitative inheritance in grasses. Proc $6^{\text {th }}$ Int Grassland Cong $1: 277-283$

Constable GA, Hearn AB (1978) Agronomic and physiological response of soybean and sorghum crops to water deficit. Aus J Plant Physiol 5:159-167

Deepika C, Lal SK, Talukdar A, Mandal B, Prachi Yadav, Singh KP, Sunil Kumar (2017) Screening and identification of resistant sources against Cowpea mild mottle virus (CPMMV) disease in soybean. Indian J. Genet. 77(2): 287292

Federer WT (1956) Augmented designs. Hawaii Planters Record 55:191-208

Frahm MA, Rosas JC, Mayek-Perez N, Lopez-Salinas E, Acosta-Gallegos JA, Kelly JD (2004) Breeding beans for resistance to terminal drought in the lowland tropics. Euphytica 136: 223 232.

Gangopadhyay KK, Singh A, Bag MK, Ranjan P, Prasad TV, Roy A and Dutta M (2017) Diversity analysis and evaluation of wild Abelmoschus species for agro-morphological traits and major biotic stresses under the north western agro-climatic condition of India. Genet. Resour. Crop Evol 64(4): 775-790.

Hirasawa T, Tanaka K, Miyamoto D, Takai M, Ishihara K (1994) Effects of preflowering soil moisture deficit on dry matter production and eco-physiological characteristics in soybean plants under drought conditions during grain filling. Japan J Crop Sci 63:721-730

Iqbal Z, Arshad M, Ashraf M, Mahmood T, Waheed A (2008) Evaluation of soybean (Glycine max (L.) Merrill) germplasm for some important morphological traits using multivariate analysis. Pak. J. Bot. 40(6):2323-2328

Joshi OP, Bhatia VS (2003) Stress management in soybean. Singh $\mathrm{H}$ and Hegde DM (eds) Souvenir, National seminar on stress management in oilseeds for attaining self-reliance in vegetable oils. Indian Soc. Oilseeds Res. Hyderabad, India.13-25

Kargar SMA, Mostafaie A, Hervan EM, Pourdad SS (2015) Study on genetic variation of 14 soybean cultivars using cluster and factor analysis under water stress and nonstress conditions. J Bio \& Env Sci 6(3):100-112

$\mathrm{Ku}$ YS, Au-Yeung WK, Yung YL, Li MW, Wen CQ, Liu X, Lam HM (2013) Drought stress and tolerance. In: J. E. Board (ed.), Soybean A Comprehensive Survey of International Soybean Research Genetics, Physiology, Agronomy and Nitrogen Relationships, 209-237. InTech, New York.

Liu K (1997). Soybeans: Chemistry, Technology and Utilization, Aspen Publishers

Mahbub MM, Mamunur Rahman M, Hossain MS, Nahar L, Shirazy BJ (2016) Morpho-physiological variation in soybean (Glycine $\max$ (L.) Merrill). Am-Euras J Agric \& Environ Sci 16 (2):234-238

Manavalan LP, Guttikonda SK, Tran LP, Nguyen HT (2009) Physiological and molecular approaches to improve drought resistance in soybean. Plant and Cell Physiol 50(7):1260-1276

Nicolas ME, Turner NC (1993) Use of chemical desiccants and senescing agent to select wheat lines maintaining stable grain size during post anthesis drought. Field Crop Res 31:155-171

Regan KL, Whan BR, Turner NC (1993) Evaluation of chemical desiccant as a selection technique for drought resistance in a dry land wheat breeding programme. Aus J Agric Res 44:16831691 
Royo C, Blanco R (1998) Use of potassium iodide to mimic drought stress in triticale. Field Crop Res 59:201-212

Saitoh K, Mahmood T, Kurada T (1999) Effect of moisture stress at different growth stages on flowering and pod set in determinate and indeterminate soybean cultivars. Japan J Crop Sci 68:537-544

Sawhney V, Singh DP (2002) Effects of chemical desiccation at the post anthesis stage on some physiological and biochemical changes in the flag leaf of contrasting wheat genotypes. Field Crop Res 77:1-6

Schnyder H (1993) The role of carbohydrate storage and redistribution in the sourcesink relations of wheat and barley during grain filling-a review. New Phytologist 123:233-245

Shannon CE, Weaver W (1949) The mathematical theory of communication. University of Illinois Press, Urbana.

Sionit N, Kramer PJ (1977) Effect of water stress during different stages of growth of soybean. Agron J 69:274-278

Siddique KHM, Regan KL, Tennant D, Thomson BD (2001) Water use and water use efficiency of cool season grain legumes in low rainfall Mediterranean-type environments. European J Agron 15(4): 267-280

Singh AK, Singh A, Singh AK, Shamim M, Vikram P, Singh S, Chaturvedi G (2012) Application of potassium iodide as a new agent for screening of drought tolerance upland rice genotypes at flowering stage. Plant Knowledge J. $1: 25-32$

Subbarao GV, Johansen C, Slinkard AE, Nageswara Rao RC, Saxena NP, Chauhan YS (1995) Strategies for improving drought tolerance in grain legumes. Crit Rev Plant Sci 14:469-523

Upadhyaya HD, Ortiz R, Paula J. Bramel, Sube Singh (2001) Phenotypic diversity for morphological and agronomic characteristics in chickpea core collection. Euphytica 123: 333-342, 2002.

Wilson RF (2004) Seed Composition in Soybeans: Improvement, Production, and Uses. Agronomy Monographs $3^{\text {rd }}$ eds. HR Boerma and J E Specht eds. ASA-CSSA-SSSA, Madison, WI, USA. $621-677$

\section{How to cite this article:}

Sreenivasa, V., S.K. Lal, A. Talukdar, P. Kiran Babu, H.K. Mahadeva Swamy, Darsing R. Rathod, Raju R. Yadav, Shatakashi Poonia, K.V. Bhat and Viswanathan, C. 2019. Evaluation of Soybean Germplasm Lines for Agro-Morphological Traits and Terminal Drought Tolerance. Int.J.Curr.Microbiol.App.Sci. 8(04): 105-127. doi: https://doi.org/10.20546/ijcmas.2019.804.013 